\title{
Diversidad de mamíferos en un remanente de bosque urbano de la ciudad de Medellín (Antioquia, Colombia)
}

\author{
Mammal diversity in an urban forest remnant located in the city of Medellín, (Antioquia, \\ Colombia)
}

Tomás Villada-Cadavid ${ }^{1}$, Iván Darío Soto-Calderón ${ }^{2 *}$

\begin{abstract}
Resumen
La gran diversidad de especies en centros urbanos de Colombia contrasta con la rápida e intensa degradación de los hábitats donde se concentra dicha diversidad. Dada la importancia de estos entornos para la conservación biológica urbana y la creciente presión que sobre ellos existe, se estudió la composición de la comunidad de mamíferos en la cuenca de la quebrada La Gómez, en la densamente poblada ciudad de Medellín, y se presentaron recomendaciones para su conservación. Esta cuenca sobresale por la presencia del mono tití gris (Saguinus leucopus), uno de los pocos primates urbanos de Colombia. Se lograron identificar cuatro especies de murciélago, una ardilla, una zarigüeya, una comadreja y el mono tití gris. Resalta la gran abundancia de zarigüeyas, posiblemente asociada con la alta disponibilidad de recursos de comida. Esto contrasta con una pobre diversidad de roedores nativos, quizá como consecuencia de la depredación por los numerosos gatos de la zona. Estas especies presentan dietas generalistas, aprovechan recursos antropogénicos de comida o tienen alta capacidad de dispersión, propiedades que les pueden facilitar su adaptación a hábitats aislados en parches urbanos de bosque. Todas estas especies tienen importantes roles ecológicos como la polinización, control poblacional de artrópodos y vertebrados y dispersión de especies vegetales. Sugerimos 1) mejorar las técnicas de disposición de residuos y compostaje para evitar conflictos con zarigüeyas, 2) realizar control poblacional de gatos ferales y concientizar sobre la tenencia responsable de gatos y otras mascotas, 3) sembrar especies vegetales nativas que sirvan de sostén a la fauna de la zona, y 4) emprender campañas de limpieza de la quebrada y disposición responsable de residuos domésticos.
\end{abstract}

Palabras claves: conservación, Chiroptera, fauna urbana, marsupial, primates, Rodentia

\begin{abstract}
The great diversity of species in urban centers in Colombia contrasts with the rapid and intense degradation of the habitats where this diversity occurs. Given the importance of these environments for urban biological conservation and the increasing pressure on them, we studied the composition of the mammal community in the basin of La Gómez creek, located in the densely populated city of Medellin, and provided recommendations for its conservation. This basin stands out for the presence of the white-footed tamarin (Saguinus leucopus), one of the few urban primates in Colombia. We identified four species of bats, a squirrel, an opossum, a weasel, and the white-footed tamarin. Particularly striking is the great abundance of opossums, possibly associated with high availability of food resources. This contrasts with a poor diversity of native rodents, perhaps as a result of predation by the many domestic cats in this area. These species have generalist diets, take advantage of anthropogenic food resources or have high dispersal capacity, properties that can facil-
\end{abstract}

\footnotetext{
1. Grupo de investigación de Mastozoología, Universidad de Antioquia, Medellín, Colombia.

2. Laboratorio de Genética Animal, Grupo GAMMA, Universidad de Antioquia, Medellín, Colombia.

* Autor de correspondencia: <ivan.soto@udea.edu.co>
} 
itate their adaptation to geographically isolated habitats in urban forest patches. All these species have important ecological roles such as pollination, population control of arthropods and vertebrates, and dispersal of plant species. We suggest to 1) improve waste disposal and composting techniques to avoid conflicts with opossums, 2) carry out population control of feral cats and raise awareness about the responsible possession of cats and other pets, 3) sow native plant species that support local wildlife, and 4) undertake cleaning campaigns on the creek and responsible disposal of household waste.

Keywords: Conservation, Chiroptera, marsupial, primates, Rodentia, urban fauna

\section{INTRODUCCIÓN}

En Colombia se registran 528 especies de mamíferos (Sistema de Información sobre Biodiversidad de Colombia, 2020), representadas en 14 órdenes y alrededor de 49 familias (Ramírez-Chaves, Suarez-Castro, y González-Maya, 2016). El grupo de mamíferos más diverso para el país es el de los murciélagos (orden Chiroptera) con alrededor de 205 especies, seguido de los roedores (orden Rodentia) con unas 132 especies; aunque estas cifras están siendo constantemente modificadas debido a avances en el conocimiento de la diversidad del país (Ramírez-Chaves y SuárezCastro, 2014; Ramírez-Chaves et al., 2016; Solari et al., 2013).

Una zona altamente intervenida y densamente poblada, como lo es el área metropolitana del Valle de Aburrá (AMVA), presenta importantes remanentes de bosque en sus laderas y en parches de bosque dentro del área urbana donde se han encontrado cerca del 100 especies de mamíferos silvestres nativos en 35 familias y diez órdenes, cerca del $10 \%$ de las especies registradas para todo el territorio nacional (Área Metropolitana del Valle de Aburrá, 2015). A pesar de los avances en los listados de especies de mamíferos, se considera que aún falta por conocer parte de la diversidad biológica de la región, bache que se irá completando en la medida que se continúe con esfuerzos de investigación y divulgación de la información, lo que se constituye en insumo para la integración de la biodiversidad a planes de manejo y ordenamiento territorial y para la apropiación comunitaria de los recursos biológicos.

Los parches de bosque asociados a las fajas de retiro de las corrientes naturales de agua son algunos de los escasos hábitats para especies de mamíferos y otros vertebrados dentro de las matrices urbanas.
Además de su valor para la conservación de estas especies nativas, los retiros en cuencas de ríos y quebradas urbanas prestan servicios ecosistémicos como regulación del agua de escorrentía, purificación del aire y regulación microclimática, al igual que confieren valiosas oportunidades para el esparcimiento de las comunidades locales, la educación ambiental y la investigación (Montoya et al., 2019). Dada su importancia para la conservación del recurso hídrico, estas zonas además suelen estar protegidas por la normatividad dentro de los planes de ordenamiento territorial y particularmente, en la ciudad de Medellín, se consideran como áreas de interés estratégico dentro de la estructura ecológica (Alcaldía de Medellín. Departamento Administrativo de Planeación, 2018). Una de estas franjas de bosque se encuentra en la quebrada La Gómez, al noroccidente de la ciudad de Medellín (Antioquia, Colombia). Esta representa uno de los escasos entornos urbanos donde aún prevalece el mono tití gris (Saguinus leucopus, Günther, 1877) en el área metropolitana, especie nativa del noroccidente colombiano y actualmente en peligro de Extinción (EN) de acuerdo con los parámetros de la Unión Internacional para la Conservación de la Naturaleza (Morales-Jiménez, Link, y Stevenson, 2008). Lamentablemente, la cuenca de esta quebrada está aislada de otras zonas boscosas del Valle de Aburrá, por lo que se prevé una diversidad de especies pequeña en relación con lo reportado previamente para el Valle de Aburrá. En este trabajo se evaluó la diversidad de especies de mamíferos terrestres y voladores, y se estimó el número y composición de grupos sociales de tití gris presentes en la cuenca de la quebrada La Gómez, en el municipio de Medellín.

\section{MATERIALES Y MÉTODOS}

La quebrada La Gómez se encuentra ubicada en la comuna siete, también denominada Robledo, del 
municipio de Medellín, Valle de Aburrá, al norte de la cordillera Central colombiana $\left(6^{\circ} 16^{\prime} 7,777^{\prime \prime} \mathrm{N}\right.$ y $\left.75^{\circ} 35^{\prime} 30,501^{\prime \prime} \mathrm{W}\right)$. Los muestreos se realizaron en remanentes de bosque ribereño, que corresponden a bosque húmedo premontano (bh-P) (Espinal, 1985), a lo largo de un tramo de $1,1 \mathrm{Km}$ que hacen parte del retiro de la quebrada (figura 1). El cuerpo de agua se encuentra intervenido a lo largo del área de estudio, con vertimientos de aguas provenientes de zonas residenciales aledañas.

En diciembre de 2017 se estudió la composición de la comunidad de mamíferos empleando diferentes metodologías, incluyendo trampas Sherman® para pequeños mamíferos terrestres, trampas cámaras para mamíferos no voladores, recorridos diurnos por la zona e instalación de redes de niebla para murciélagos (Hoffmann et al., 2010; Voss y Emmons, 1996). Toda la información obtenida en campo se consignó en libretas de campo, incluyendo fecha y hora de cada registro o captura, la especie, el tipo de trampa, medidas morfométricas de los individuos muestreados y estado reproductivo.

\section{Captura de pequeños mamíferos no voladores}

Para el muestreo de pequeños mamíferos terrestres se instalaron 40 trampas Sherman ${ }^{\circledR}$ a nivel del suelo (cerca de refugios, entre la hojarasca, matorrales, bajo rocas y cerca de cursos de agua) y en ramas ubicadas entre uno y dos metros de altura, dispuestas en forma de transectos, ubicadas aproximadamente cada $15 \mathrm{~m}$ en 20 estaciones con dos trampas por estación, durante ocho noches consecutivas. Se realizó un esfuerzo de muestreo total de 320 trampasnoche durante el mes de diciembre 2017, cebando las trampas con una mezcla de avena, mantequilla de maní y esencia de vainilla durante las primeras cuatro noches, y con una mezcla de atún y avena durante las siguientes cuatro noches (tabla 1). Las trampas se revisaron y recebaron a diario en las horas de la mañana. Los animales capturados se transportaron en bolsas de tela para su identificación en campo mediante el uso de las claves pertinentes hasta el nivel taxonómico que estas lo permitieron (género o especie) (Gardner, 2007; Patton, Pardiñas, y Dt'Elía, 2015).

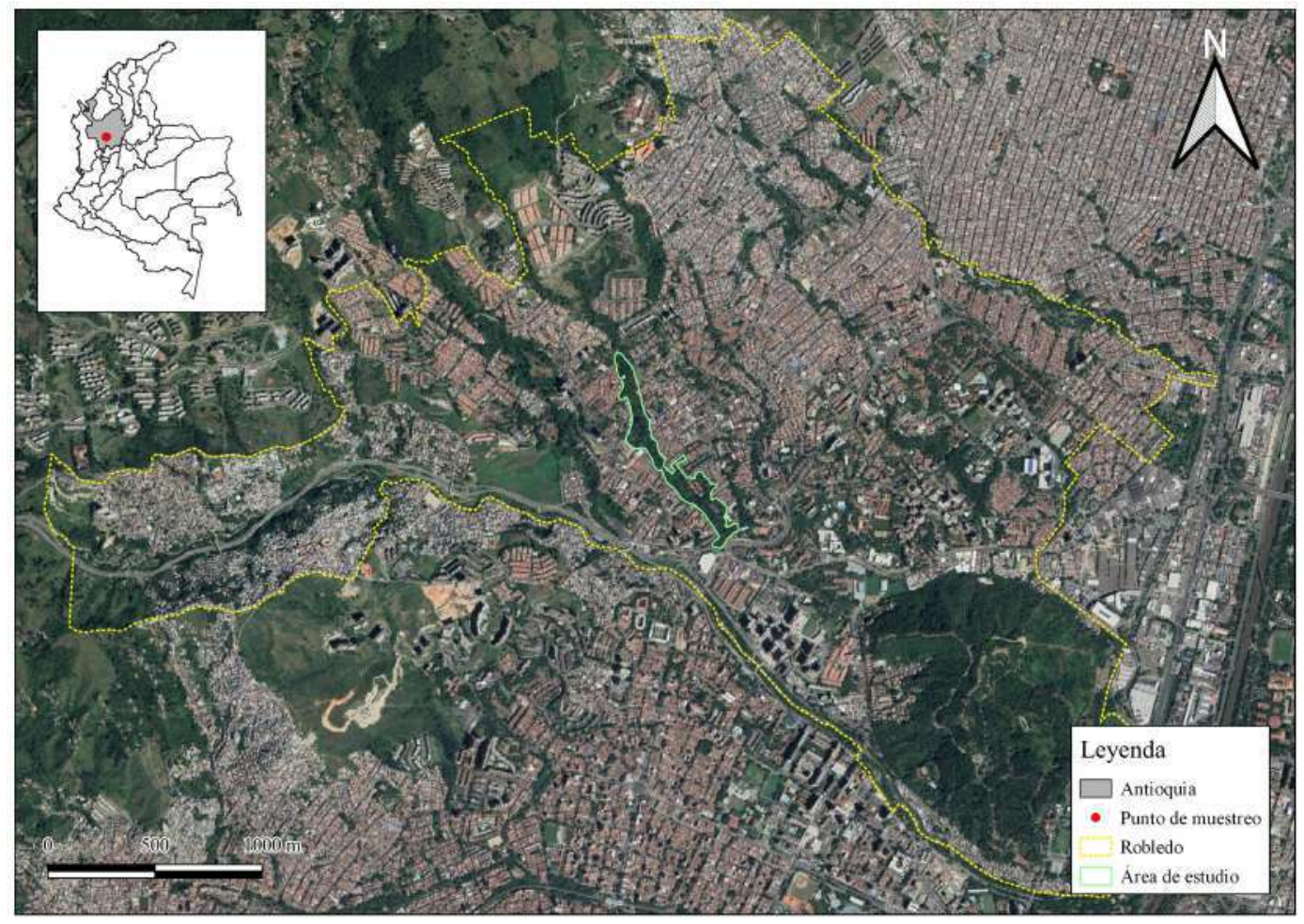

Figura 1. Ubicación del área de estudio en el sector de Robledo, ciudad de Medellín. 
Tabla 1. Coordenadas de las estaciones donde se instalaron trampas Sherman ${ }^{\circledR}$, redes de niebla y cámaras trampa.

\begin{tabular}{|c|c|c|c|c|}
\hline Método & Latitud & Longitud & Altura (m s. n. m.) & Observación \\
\hline \multirow{20}{*}{ Trampa Sherman ${ }^{\circledR}$} & $6^{\circ} 16^{\prime} 30,00^{\prime \prime} \mathrm{N}$ & $75^{\circ} 35^{\prime} 36,31^{\prime \prime} \mathrm{W}$ & 1555 & Estación 1 \\
\hline & $6^{\circ} 16^{\prime} 29,86^{\prime \prime} \mathrm{N}$ & $75^{\circ} 35^{\prime} 36,74^{\prime \prime} \mathrm{W}$ & 1555 & Estación 2 \\
\hline & $6^{\circ} 16^{\prime} 30,54^{\prime \prime} \mathrm{N}$ & $75^{\circ} 35^{\prime} 36,64^{\prime \prime} \mathrm{W}$ & 1557 & Estación 3 \\
\hline & $6^{\circ} 16^{\prime} 31,19^{\prime \prime} \mathrm{N}$ & $75^{\circ} 35^{\prime} 37,57^{\prime \prime} \mathrm{W}$ & 1561 & Estación 4 \\
\hline & $6^{\circ} 16^{\prime} 31,33^{\prime \prime} \mathrm{N}$ & $75^{\circ} 35^{\prime} 37,39^{\prime \prime} \mathrm{W}$ & 1561 & Estación 5 \\
\hline & $6^{\circ} 16^{\prime} 31,48^{\prime \prime} \mathrm{N}$ & $75^{\circ} 35^{\prime} 37,68^{\prime \prime} \mathrm{W}$ & 1562 & Estación 6 \\
\hline & $6^{\circ} 16^{\prime} 32,48^{\prime \prime} \mathrm{N}$ & $75^{\circ} 35^{\prime} 39,30^{\prime \prime} \mathrm{W}$ & 1566 & Estación 7 \\
\hline & $6^{\circ} 16^{\prime} 33,17^{\prime \prime} \mathrm{N}$ & $75^{\circ} 35^{\prime} 40,06^{\prime \prime} \mathrm{W}$ & 1570 & Estación 8 \\
\hline & $6^{\circ} 16^{\prime} 33,53^{\prime \prime} \mathrm{N}$ & $75^{\circ} 35^{\prime} 40,70^{\prime \prime} \mathrm{W}$ & 1574 & Estación 9 \\
\hline & $6^{\circ} 16^{\prime} 34,07^{\prime \prime} \mathrm{N}$ & $75^{\circ} 35^{\prime} 40,56^{\prime \prime} \mathrm{W}$ & 1575 & Estación 10 \\
\hline & $6^{\circ} 16^{\prime} 34,97^{\prime \prime} \mathrm{N}$ & $75^{\circ} 35^{\prime} 41,50^{\prime \prime} \mathrm{W}$ & 1581 & Estación 11 \\
\hline & $6^{\circ} 16^{\prime} 34,68^{\prime \prime} \mathrm{N}$ & $75^{\circ} 35^{\prime} 41,17^{\prime \prime} \mathrm{W}$ & 1579 & Estación 12 \\
\hline & $6^{\circ} 16^{\prime} 35,15^{\prime \prime} \mathrm{N}$ & $75^{\circ} 35^{\prime} 40,99^{\prime \prime} \mathrm{W}$ & 1580 & Estación 13 \\
\hline & $6^{\circ} 16^{\prime} 34,18^{\prime \prime} \mathrm{N}$ & $75^{\circ} 35^{\prime} 42,14^{\prime \prime} \mathrm{W}$ & 1580 & Estación 14 \\
\hline & $6^{\circ} 16^{\prime} 38,03^{\prime \prime} \mathrm{N}$ & $75^{\circ} 35^{\prime} 44,27^{\prime \prime} \mathrm{W}$ & 1596 & Estación 15 \\
\hline & $6^{\circ} 16^{\prime} 38,96^{\prime \prime} \mathrm{N}$ & $75^{\circ} 35^{\prime} 45,02^{\prime \prime} \mathrm{W}$ & 1602 & Estación 16 \\
\hline & $6^{\circ} 16^{\prime} 39,07^{\prime \prime} \mathrm{N}$ & $75^{\circ} 35^{\prime} 45,53^{\prime \prime} \mathrm{W}$ & 1604 & Estación 17 \\
\hline & $6^{\circ} 16^{\prime} 39,18^{\prime \prime} \mathrm{N}$ & $75^{\circ} 35^{\prime} 44,41^{\prime \prime} \mathrm{W}$ & 1601 & Estación 18 \\
\hline & $6^{\circ} 16^{\prime} 39,54^{\prime \prime} \mathrm{N}$ & $75^{\circ} 35^{\prime} 45,10^{\prime \prime} \mathrm{W}$ & 1604 & Estación 19 \\
\hline & $6^{\circ} 16^{\prime} 40,12^{\prime \prime} \mathrm{N}$ & $75^{\circ} 35^{\prime} 45,49^{\prime \prime} \mathrm{W}$ & 1608 & Estación 20 \\
\hline \multirow{8}{*}{ Red de niebla } & $6^{\circ} 16^{\prime} 31,69^{\prime \prime} \mathrm{N}$ & $75^{\circ} 35^{\prime} 37,79^{\prime \prime} \mathrm{W}$ & 1563 & Noche 1 \\
\hline & $6^{\circ} 16^{\prime} 30,72^{\prime \prime} \mathrm{N}$ & $75^{\circ} 35^{\prime} 35,52^{\prime \prime} \mathrm{W}$ & 1556 & Noche 2 \\
\hline & $6^{\circ} 16^{\prime} 32,92^{\prime \prime} \mathrm{N}$ & $75^{\circ} 35^{\prime} 37,93^{\prime \prime} \mathrm{W}$ & 1562 & Noche 3 \\
\hline & $6^{\circ} 16^{\prime} 34,39^{\prime \prime} \mathrm{N}$ & $75^{\circ} 35^{\prime} 40,85^{\prime \prime} \mathrm{W}$ & 1577 & Noche 4 \\
\hline & $6^{\circ} 16^{\prime} 52,61^{\prime \prime} \mathrm{N}$ & $75^{\circ} 35^{\prime} 48,62^{\prime \prime} \mathrm{W}$ & 1659 & Noche 5 \\
\hline & $6^{\circ} 16^{\prime} 58,66^{\prime \prime} \mathrm{N}$ & $75^{\circ} 35^{\prime} 51,43^{\prime \prime} \mathrm{W}$ & 1680 & Noche 6 \\
\hline & $6^{\circ} 16^{\prime} 55,56^{\prime \prime} \mathrm{N}$ & $75^{\circ} 35^{\prime} 48,98^{\prime \prime} \mathrm{W}$ & 1669 & Noche 7 \\
\hline & $6^{\circ} 17^{\prime} 00,24^{\prime \prime} \mathrm{N}$ & $75^{\circ} 35^{\prime} 53,92^{\prime \prime} \mathrm{W}$ & 1690 & Noche 8 \\
\hline \multirow{4}{*}{ Cámara Trampa } & $6^{\circ} 16^{\prime} 30,54^{\prime \prime} \mathrm{N}$ & $75^{\circ} 35^{\prime} 36,64^{\prime \prime} \mathrm{W}$ & 1557 & Cámara 1: 8 noches \\
\hline & $6^{\circ} 16^{\prime} 32,92^{\prime \prime} \mathrm{N}$ & $75^{\circ} 35^{\prime} 40,56^{\prime \prime} \mathrm{W}$ & 1571 & Cámara 2: 8 noches \\
\hline & $6^{\circ} 16^{\prime} 34,72^{\prime \prime} \mathrm{N}$ & $75^{\circ} 35^{\prime} 42,07^{\prime \prime} \mathrm{W}$ & 1581 & Cámara 3: 34 noches \\
\hline & $6^{\circ} 16^{\prime} 52,46^{\prime \prime} \mathrm{N}$ & $75^{\circ} 35^{\prime} 50,60^{\prime \prime} \mathrm{W}$ & 1663 & Cámara 4: 34 noches \\
\hline
\end{tabular}

\section{Registros de mamíferos medianos no voladores}

Se instalaron cuatro cámaras trampa con un esfuerzo acumulado de muestreo de 84 días-cámara. Estas se fijaron a árboles aproximadamente a $50 \mathrm{~cm}$ del suelo, ubicadas en sitios donde se encontraron rastros como huellas y excrementos (Rovero, Tobler, y Sanderson, 2010) (tabla 1). Adicionalmente, se realizaron recorridos diurnos diarios en las horas de la mañana, mientras se revisaban y cebaban las tramas Sherman®. Estos recorridos tuvieron una duración de al menos una hora entre las 10:00 y 12:00 h, durante ocho días consecutivos. Durante los recorridos se consignaron las especies registradas visualmente o mediante rastros, huellas o restos. Por razones de seguridad no se realizaron recorridos nocturnos.

\section{Muestreo de murciélagos}

Para el muestreo de quirópteros se usaron cuatro redes de niebla de $12 \times 3 \mathrm{~m}$. Estas redes se ubicaron a nivel del suelo entre los cero y tres metros de altura, teniendo en cuenta áreas óptimas para el vuelo o forrajeo de los murciélagos como son: bordes y claros de bosque, corredores naturales, quebradas y cuerpos de agua, trochas y plantas con frutos o flores usadas como alimento. Las redes se desplegaron desde las 18:00 horas hasta las 22:00 h, rango en el cual se presentan los picos de actividad de este grupo (Aguiar y Marinho-Filho, 2004). Cada red de niebla se revisó periódicamente cada 30 min o con mayor frecuencia, dependiendo de la actividad que se presentara en el sitio. Se realizaron muestreos de murciélagos durante ocho noches en diferentes puntos ubicados a lo largo 
de la cuenca de la quebrada (tabla 1), acumulando un esfuerzo total de muestreo de 32 horas-noche.

Los animales capturados se transportaron en bolsas de tela para su determinación taxonómica en campo mediante el uso de las claves pertinentes hasta el nivel taxonómico que estas lo permitieron, $i$. e. género o especie (Díaz, Solari, Aguirre, Aguiar, y Barquez, 2016; Gardner, 2007). Cuando fue posible identificar un espécimen por sus características morfológicas externas, este fue liberado en la misma cobertura donde fue capturado. Debido a dificultades en la determinación taxonómica en campo, fue necesario recolectar un espécimen de murciélago del género Glossophaga, de modo que se pudiese realizar la determinación en el laboratorio, siguiendo caracteres dentales y craneales (Código en la Colección Teriológica de la Universidad de Antioquia CTUA-4822). El individuo recolectado fue sacrificado con una inyección letal de Roxicaína al $2 \%$ en el corazón, y posteriormente, se preservó en alcohol al $96 \%$ para evitar su descomposición (Sikes y Gannon, 2011). La recolecta de este espécimen de Glossophaga sp. estuvo amparada por el Permiso Marco de Recolección No. 0524 del 27 de mayo de 2014, otorgado a la Universidad de Antioquia por la Autoridad Nacional de Licencias Ambientales.

\section{Riqueza de especies de murciélagos}

Para los muestreos de murciélagos mediante redes de niebla se estimó la riqueza de especies y se construyó la curva de acumulación de especies con el programa EstimateS v.9 (Colwell, 2005). Las curvas de acumulación son gráficas que ilustran la cantidad de especies que se están observando en relación con una medida de esfuerzo de muestreo. Este tipo de gráficos, acompañados del cálculo de estimadores de riqueza, permiten evaluar la representatividad del muestreo realizado. Para determinar el número de especies esperadas en el sitio de estudio se computaron los estimadores de riqueza no paramétricos basados en abundancias Chao 1 y ACE (Abundance Coveragebased Estimator), los cuales son reconocidos como estimadores robustos (Basualdo, 2017). Únicamente se realizaron análisis de riqueza para este grupo, debido a que no se detectaron pequeños mamíferos mediante trampas Sherman ${ }^{\circledR}$ y solo una especie con cámaras trampa.

\section{Índices de diversidad en murciélagos}

Para la comunidad de murciélagos se calculó el índice de diversidad de Simpson, el cual depende de la dominancia relativa entre las diferentes especies de la comunidad. Además, se calculó el índice de Equidad de Pielou, el cual mide la proporción de la diversidad observada en relación con la diversidad máxima esperada, permitiendo así estimar la similitud en la abundancia de las diferentes especies. Todos los índices se calcularon empleando el programa PAST 3 (Hammer-Muntz, Harper, y Ryan, 2001).

\section{RESULTADOS}

\section{Riqueza y composición de especies}

No se logró capturar ningún espécimen con trampas Sherman ${ }^{\circledR}$; sin embargo, durante uno de los recorridos se encontraron huellas de roedor y la mandíbula de un marsupial del género Didelphis. Mediante el uso de redes de niebla se captuaron 48 especímenes, 35 de los cuales fueron Artibeus lituratus (Olfers, 1818), diez Glossophaga soricina (Pallas, 1766), dos Sturnira parvidens (Goldman, 1917) y un Myotis riparius (Handley, 1960). Las especies A. lituratus, $G$. soricina y $S$. parvidens pertenecen a la familia Phyllostomidae, mientras que $M$. riparius es de la familia Verpertilionidae.

Se grabaron 1806 videos en total haciendo uso de cámaras trampa; de estos, 888 contenían videos de fauna, 581 de ellos correspondientes específicamente a mamíferos. Los demás videos capturados correspondieron a aves, reptiles o falsos positivos. Mediante esta metodología se lograron identificar las especies silvestres Didelphis marsupialis (Linnaeus, 1758) y Notosciurus granatensis (Humboldt, 1811) en 541 y 8 videos, respectivamente. Se hicieron 31 registros de videos de gatos y un video de mamífero cuya especie no se pudo identificar.

Durante los recorridos diurnos se logró identificar un grupo de titíes gris ( $S$. leucopus) compuesto por 10 individuos, incluyendo dos crías, así como una zarigüeya (D. marsupialis). Durante los muestreos 
el grupo siempre permaneció unido, desplazándose en las mañanas y en las tardes. Se observó que el grupo se alimenta ocasionalmente de frutas que dejan los habitantes de las unidades residenciales circundantes en balcones y plataformas. En dirección a la cabecera de la quebrada, se encontró también un grupo de dos individuos en inmediaciones de un guadual. Durante los recorridos también se registraron varios individuos de ardillas ( $N$. granatensis) cuya abundancia no fue registrada, así como un individuo de comadreja (Mustela frenata, Lichtenstein, 1831). En uno de los recorridos se encontraron restos óseos de un presunto marsupial del género Didelphis, el cual fue llevado al laboratorio y depositado en la Colección Teriológica de la Universidad de Antioquia bajo el código CTUA-4821. Se lograron registrar en total ocho especies de mamíferos silvestres (figura 2), dentro de seis familias en los órdenes Chiroptera, Carnivora, Didelphimorphia, Primates y Rodentia.

\section{Representatividad del muestreo y diversidad de murciélagos}

En la curva de acumulación de especies de murciélagos se observa un comportamiento asintótico, lo que permite inferir que se capturaron todas o casi todas las especies de murciélagos que se encuentran en el sitio de estudio (figura 3). De acuerdo con el estimador de riqueza basado en abundancias Chao 1 se esperaban encontrar cuatro especies de murciélagos, lo que coincidió con el valor observado. Según el estimador ACE se esperaban encontrar cinco especies, según lo cual se habría observado el $80 \%$ de la riqueza esperada.

\section{Índices de diversidad en murciélagos}

En general, la comunidad de murciélagos presenta una baja riqueza de especies, con solo cuatro especies identificadas. El índice de Simpson fue 0,5773, revelando así una dominancia intermedia en la comunidad, ya que el índice toma valores de cero cuando todas las especies presentan la misma abundancia y valores de uno cuando una especie domina completamente la comunidad (tabla 2). La Equidad de Pielou fue 0,5556 , es decir un valor intermedio entre 0 y 1 , donde 1 indicaría que todas las especies son igualmente abundantes.

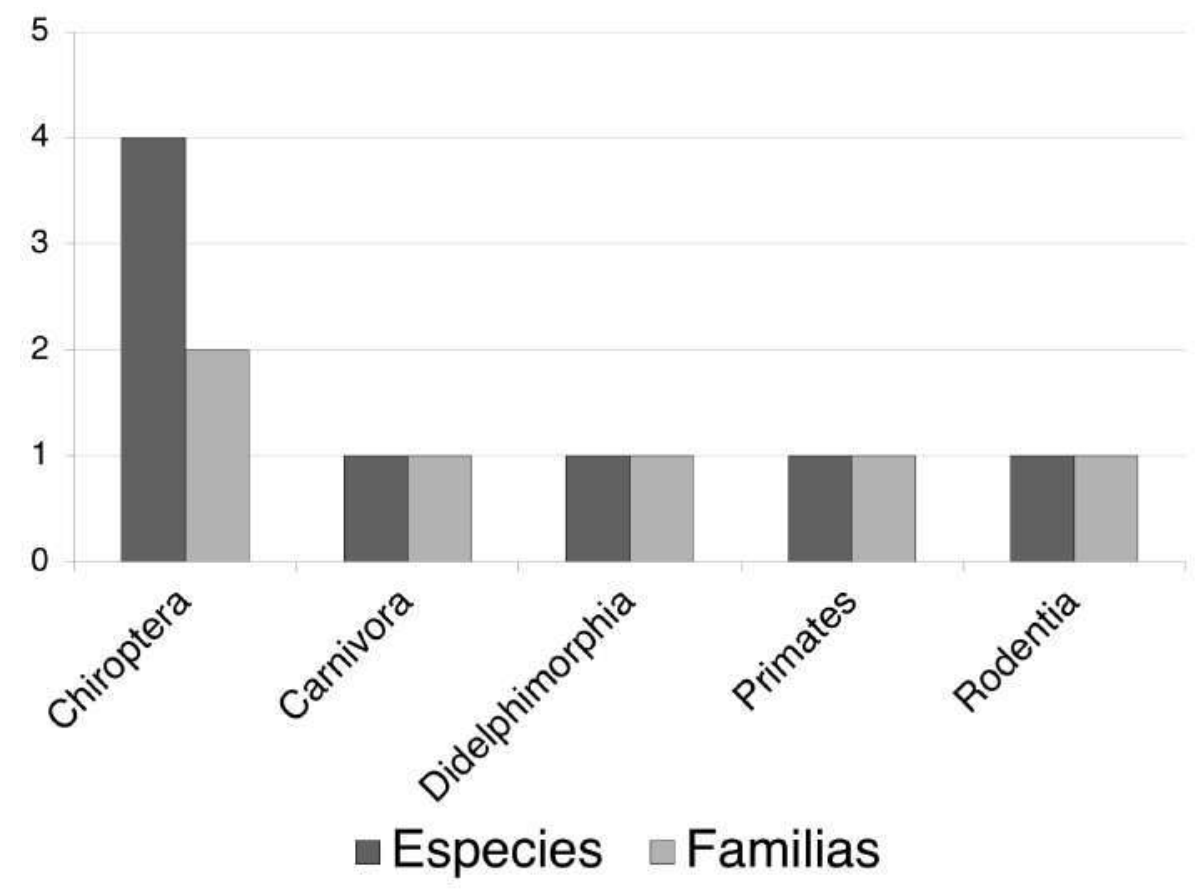

Figura 2. Representatividad de cada orden en número de especies y número de familias. 


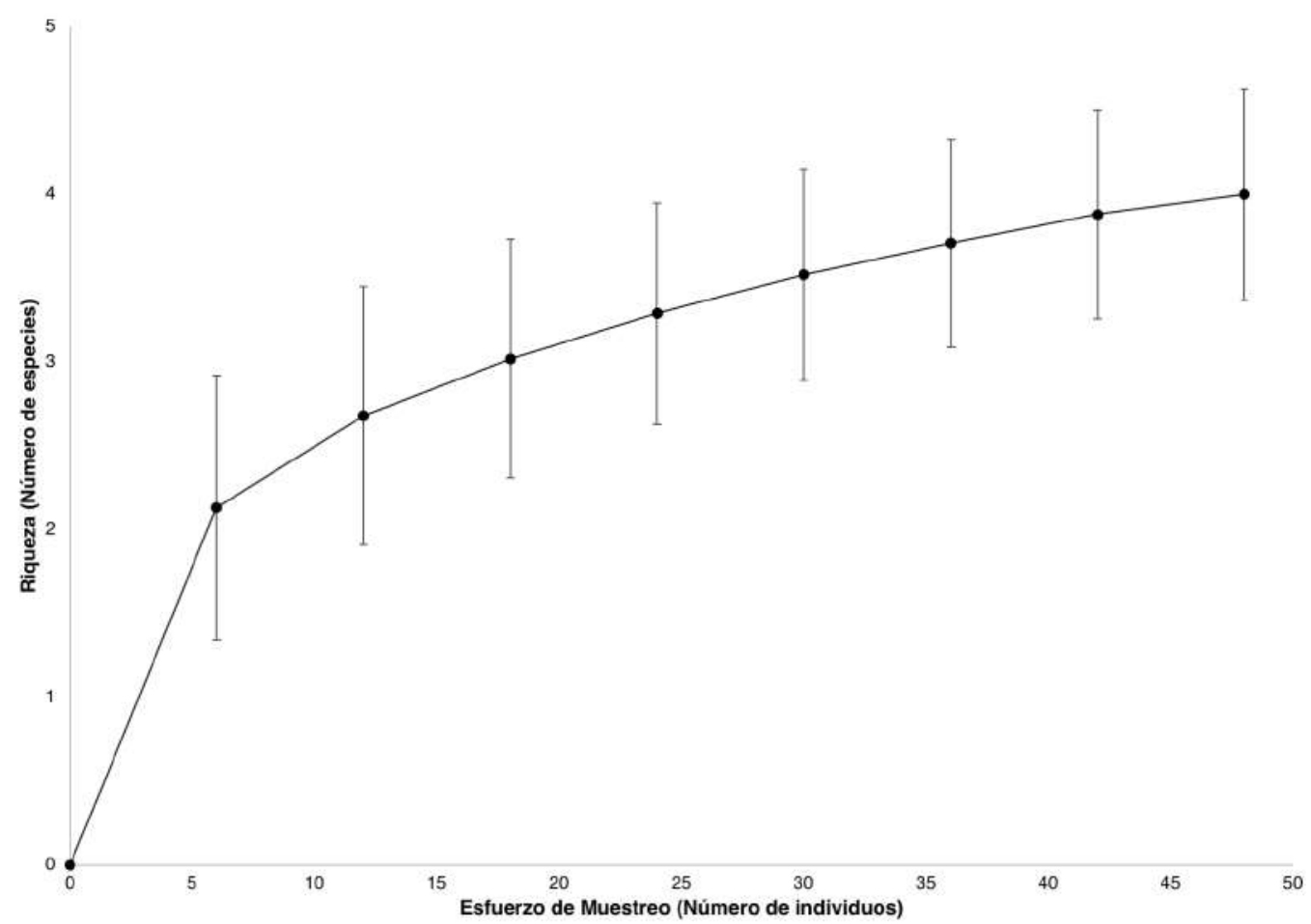

Figura 3. Curva de acumulación de especies para el orden Chiroptera.

Tabla 2. Índices de diversidad de murciélagos.

\begin{tabular}{l|l}
\hline Índice & Valor \\
\hline Especies & 4 \\
$\mathrm{~N} .{ }^{\circ}$ de individuos & 48 \\
Simpson (D) & 0,5773 \\
Shannon (H) & 0,7702 \\
Equidad (J) & 0,5556 \\
\hline
\end{tabular}

\section{DISCUSIÓN}

Debido al rápido crecimiento de las urbes, elementos como el tamaño, la calidad y la conectividad de remanentes de bosque urbano que permitan la conservación de especies nativas son cada vez más vulnerados (Delgado-V, Londoño, Saravia, y BedoyaViana, 2017). Uno de esos escasos entornos, en el casco urbano del Valle de Aburrá, es la cuenca de la quebrada La Gómez en el noroccidente de la ciudad de Medellín. Allí se logró identificar un total de ocho especies de mamíferos distribuidos en cinco familias.
Mediante el uso de cámaras trampa se registraron zarigüeyas y ardillas. Aunque corresponde a solo dos especies, este podría ser cercano al total de especies detectables mediante dicha metodología, ya que después de la tercera noche de muestreo no se registraron nuevas especies en las cámaras. El uso de redes de niebla para captura de murciélagos permitió detectar cuatro especies con distintos hábitos alimenticios. Los estimadores Chao 1 y ACE indicaron que el muestreo de este grupo fue bastante completo. Sin embargo, podría existir un sesgo en la representatividad de la diversidad de murciélagos de la zona debido al método de muestreo, ya que existen grupos de murciélagos, como los insectívoros, que suelen forrajear en el dosel y son difíciles de detectar en redes de niebla instaladas en estratos inferiores del bosque, tales como, Molossus bondae (Allen, 1904) y M. molossus (Pallas, 1766), por lo que se sugiere el uso de metodologías complementarias, como redes de niebla de dosel y monitoreos acústicos (Kalko y Handley, 2001). Además, otras especies de murciélagos frugívoros, como Anoura geoffroyi 
(Gray, 1838), Carollia brevicauda (Wied-Neuwied, 1821), C. perspicillata (Linnaeus, 1758) y Dermanura bogotensis (Andersen, 1906), también podrían estar presentes en el área ya que son comunes y ya han sido reportadas ampliamente en el Valle de Aburrá (Sánchez-Londoño, Marín-C, Botero-Cañola y Solari, 2014).

Si bien no se logró la captura de ningún mamífero mediante el uso de trampas Sherman ${ }^{\circledR}$, este fenómeno pudo deberse al bajo éxito de captura registrado para este tipo de trampas, donde usualmente menos del $10 \%$ de todas las trampas activas cada noche suelen ser exitosas (Voss y Emmons, 1996). También es posible que no se hayan registrado algunas especies previamente reportadas en la región, debido a su comportamiento, uso de hábitat, dieta, tamaño corporal y uso del estrato vertical. Cabe resaltar que solo una especie de roedor Cricetidae de distribución natural para la región (Zygodontomys brunneus, Thomas, 1898) ha sido reportada previamente en la zona urbana del Valle de Aburrá (Lambert, Malcolm, y Zimmerman, 2005; Sánchez-Londoño et al., 2014). Finalmente, los recorridos diurnos permitieron identificar tres tipos de mamíferos, incluyendo dos no detectados con los demás métodos, como son el tití gris y la comadreja. A pesar de las posibles limitaciones en los métodos empleados, estos fueron complementarios y llevaron a la detección de especies de mamíferos propias del Valle de Aburrá, que ya han sido identificadas en otros puntos del área urbana (Sánchez-Londoño et al., 2014).

La mayoría de las especies registradas presentan amplios rangos de distribución en Colombia (Solari et al., 2013). Estas son especies resilientes a la perturbación o con dietas generalistas que les permiten aprovechar los recursos disponibles en ambientes intervenidos (Cabrera-Jaramillo, Galeano-Román, Mazabel-Riera, Quintana-Diosa, y Monsalve-Buriticá, 2017; Sánchez-Londoño et al., 2014). Además, grupos con alta capacidad de dispersión como los murciélagos pueden eludir los altos niveles de fragmentación y degradación de los remanentes de hábitat urbano y acceder a recursos por fuera de ellos. Desde esta perspectiva, el restablecimiento de la conectividad entre parches de hábitat, ya sea mediante pasos de fauna o transloca- ción de fauna, son importantes no solo para facilitar la dispersión de especies no voladoras y su acceso a los recursos, sino también para facilitar tamaños poblacionales evolutivamente viables (Lande, 1995). Esta consideración es de particular importancia en la quebrada La Gómez y en otros entornos cada vez más fragmentados del noroccidente colombiano donde se encuentran especies amenazadas como el primate S. leucopus (Morales-Jiménez et al., 2008; Soto-Calderón, Acevedo-Garcés, Álvarez-Cardona, Hernández-Castro, y García-Montoya, 2016). Una opción económica con efectos en el corto plazo son los puentes artificiales, como el instalado por el municipio de Medellín en la cuenca de la quebrada La Gómez, en febrero de 2018, para conectar dos partes de la cuenca cruzadas por una avenida de alto flujo vehicular (Hora 13 Noticias, 2018).

Dentro de las especies mejor adaptadas al bosque ribereño de la quebrada La Gómez está D. marsupialis. Aunque su detección mediante cámaras trampa fue bastante común, no se lograron distinguir individuos mediante videos, lo que impidió hacer estimativos de abundancia. Sin embargo, su alta recurrencia en las cámaras trampa presupone su gran abundancia y dominancia dentro de los mamíferos en este tipo de hábitats. Es posible que haya una amplia disponibilidad de alimento en la zona propiciada por composteras y la oferta domiciliaria de comida, ya sea esta intencional o no.

La abundancia de zarigüeyas y murciélagos contrasta con la ausencia casi total de roedores nativos. El éxito nulo de captura que tuvieron las trampas Sherman ${ }^{\circledR}$ y la baja diversidad de roedores podrían deberse, al menos en parte, a la disponibilidad de comida en focos permanentes de alimentación como los cebaderos o comederos, lo que disminuiría el atractivo del cebo colocado en las trampas instaladas. Por último, la baja diversidad de roedores puede ser un hecho real propiciado por la depredación y el ahuyentamiento que ejercen los abundantes gatos de la zona (Baker, Bentley, Ansell, y Harris 2005; May, 1988). Los gatos se desplazan libremente a lo largo de la cuenca de la quebrada y podrían contribuir a diezmar la abundancia y diversidad de las comunidades de roedores y otros pequeños vertebrados terrestres. Esta problemática se ha visto en otras áreas urbanas 
del país (Delgado-V et al., 2017; Jiménez-Alvarado et al., 2017), por lo que se recomienda evaluar dicho fenómeno y de ser necesario, proceder a realizar un control para gatos ferales de la zona y campañas educativas para concientizar a las personas sobre el manejo responsable de sus mascotas (Proulx, 1988).

Aunque las diferentes especies pueden prestar un abanico de servicios ecosistémicos, su valor es comúnmente menospreciado en entornos urbanos. No obstante, murciélagos insectívoros como $M$. riparius juegan, de hecho, un papel muy importante en el control de insectos (Kunz, de Torrez, Bauer, Lobova, y Fleming, 2011; Ochoa, Bevilacqua, y García, 2005). Murciélagos frugívoros como S. parvidens y A. lituratus o nectarívoros como $G$. soricina son importantes para la dispersión de semillas, el intercambio genético en la comunidad de plantas y el mantenimiento de la conectividad entre hábitats geográficamente aislados. Las ardillas ( $N$. granatensis), zarigüeyas (D. marsupialis) y titíes gris (S. leucopus) presentan hábitos omnívoros, consumiendo diferentes productos vegetales así como insectos y pequeños vertebrados (Área Metropolitana del Valle de Aburrá, 2015). Esto les otorga cierta plasticidad en los procesos de adaptación a ambientes cambiantes y un rol de relevancia en el efecto que pueden tener sobre los ecosistemas, dada su capacidad para facilitar la dispersión especies vegetales y controlar poblaciones de pequeñas especies de animales (DeMattia, Curran, y Rathcke, 2004). Dentro de las especies de mamíferos registrados en este estudio se encuentra la comadreja ( $M$. frenata), un carnívoro de gran importancia ecológica ya que contribuye a controlar los tamaños poblacionales de las especies que consume (Roemer, Gompper, y Van Valkenburgh, 2009).

Finalmente, para promover la protección del corredor biótico de la quebrada La Gómez y de las especies que allí habitan, se recomienda realizar campañas educativas para facilitar la comprensión de los roles ecológicos de las especies de fauna nativas, incentivar la convivencia con dichas especies, realizar campañas de limpieza y cuidado de la quebrada y emprender acciones intensivas de plantación de árboles nativos que propicien la aparición de otras especies nativas y la conectividad entre parches de bosque. En estas se sugiere vincular tanto a la autoridad ambiental (AM-
VA y Secretaria de Medio Ambiente de Medellín), como los comités ambientales y de convivencia de las comunidades residenciales en el área de influencia, las instituciones educativas establecidas en la zona como el Colegio Ferrini y el Colegio Sagrados Corazones y las organizaciones no gubernamentales con interés en conservación de la fauna y flora de la zona como la Corporación Ambientalista Los Cucaracheros.

\section{AGRADECIMIENTOS}

A Juliana Tabares Medina por su colaboración en la toma de datos de este proyecto. Este estudio fue financiado por la Secretaría de Medio Ambiente del municipio de Medellín (SMA), mediante la asistencia de la Corporación Parque Arví (Contrato No. 76, 2017).

\section{CONFLICTO DE INTERESES}

Los autores manifiestan no presentar conflictos de intereses.

\section{REFERENCIAS}

Aguiar, L.M.D.S., y Marinho-Filho, J. (2004). Activity patterns of nine phyllostomid bat species in a fragment of the Atlantic Forest in southeastern Brazil. Revista Brasileira de Zoologia, 21(2), 385-390. DOI:10.1590/S010181752004000200037

Alcaldía de Medellín. Departamento Administrativo de Planeación. (2018). POT: Plan de Ordenamiento Territorial - Asuntos Ambientales. Retrieved from http://www.concejodemedellin.gov.co/es/ seguimiento-plan-de-ordenamiento-territorial/ documentos?language_content_entity $=\mathrm{es}$

Área Metropolitana del Valle de Aburrá. (2015, Agosto 27). Imama, una aplicación digital con la guía de los mamíferos. Retrieved from https://www.metropol.gov.co/Paginas/Noticias/imamauna-aplicacion-digital-con-la-guia-de-los-mamiferos.aspx

Baker, P.J., Bentley, A.J., Ansell, R.J., y Harris S. (2005). Impact of predation by domestic cats Felis catus in an urban area. Mammal Review, 35, 302-312. DOI:10.1111/j.13652907.2005.00071.x

Basualdo, C.V. (2017). Choosing the best non-parametric richness estimator for benthic macroinvertebrates databases. Revista de la Sociedad Entomológica Argentina, 70, 27-38. https://www.biotaxa.org/RSEA/article/ view/24073/22755

Cabrera-Jaramillo, A., Galeano-Román, A., Mazabel-Riera, E., Quintana-Diosa, L., y Monsalve-Buriticá, S. (2017). 
Evaluación del estado actual de zarigüeyas (Didelphis marsupialis) en tres zonas del Valle de Aburrá. Journal of Agriculture and Animal Sciences, 6(1), 30-40. DOI:10.22507/jals.v6n1a3

Colwell, R.K. (2005). EstimateS: Statistical estimation of species richness and shared species from samples. Version 7.5. In: http://purl.oclc.org/estimates

Delgado-V, C.A., Londoño, H., Saravia, P.M., y Bedoya-Viana, M.M. (2017). Afectaciones a la fauna silvestre en las áreas urbanas andinas de Antioquia. En E., Quintero Vallejo, A.M., Benavides, N., Moreno, S., Gonzalez-Caro (Eds.). Bosques andinos, estado actual y retos para su conservación en Antioquia (pp. 419-438). Medellín, Colombia: Fundación Jardín Botánico de Medellín Joaquín Antonio Uribe, Programa Bosques Andinos (COSUDE).

DeMattia E.A., Curran L.M., y Rathcke B.J. (2004). Effects of small rodents and large mammals on Neotropical seeds. Ecology, 85, 2161-2170. DOI:10.1890/03-0254

Díaz, M.M., Solari, S., Aguirre, L.F., Aguiar, L., y Barquez, R.M. (2016). Clave de Identificación de los murciélagos de SudaméricaChave de identificação dos morcegos da America do Sul. Publicación Especial Nro, 2. Tucumán, Argentina: Fundación Programa de Conservación de los Murciélagos de Argentina.

Espinal, L.S. (1985). Geografía ecológica del Departamento de Antioquía (zonas de vida (formaciones vegetales) del Departamento de Antioquía). Revista Facultad Nacional de Agronomía Medellín, 38, 5-106. https://revistas.unal. edu.co/index.php/refame/article/view/28367/28728

Gardner, A.L. (Ed.). (2007). Mammals of South America, Volume 1: Marsupials, Xenarthrans, Shrews, and Bats. Chicago and London: University of Chicago Press.

Hammer-Muntz, Ø, Harper, D.A.T., y Ryan, P.D. (2001). PAST: Paleontological statistics software package for education and data analysis. Palaeontologia Electronica, 4, 9pp. https://palaeo-electronica.org/2001_1/past/ issue1_01.htm

Hoffmann, A., Decher, J., Rovero, F., Schaer, J., Voigt, C., y Wibbelt, G. (2010). Field methods and techniques for monitoring mammals. In J., Eymann, J., Degreef, C., Häuser, J.C., Monje, Y., Samyn, y D., Vanden Spiegel (Eds.). Manual on field recording techniques and protocols for all taxa biodiversity inventories and monitoring (pp. 482-529). Brussels: ABC Taxa.

Hora 13 Noticias. (2018, Febrero 21). Este es el nuevo puente para la fauna silvestre en Robledo [Video]. Retrieved from https://www.youtube.com/watch?v=6hL_QfptIyI

Jiménez-Alvarado, J.S., Moreno-Díaz, C., Alfonso, A.F., Giordano, A., Vela-Vargas, I.M., Gómez-Hoyos, D.A., y González-Maya, J.F. (2017). Ciudades biodiversas: mamíferos medianos de la Reserva Forestal Protectora Bosque Oriental de Bogotá DC, Colombia. Mammalogy Notes, 4 (1), 37-41. DOI:10.47603/manovol4n1.37-41

Kalko, E.K., y Handley, C.O. (2001). Neotropical bats in the canopy: diversity, community structure, and implications for conservation. Plant Ecology, 153, 319-333. DOI:10.1023/A:1017590007861

Kunz, T.H., de Torrez, E.B., Bauer, D., Lobova, T., y Fleming, T.H. (2011). Ecosystem services provided by bats. Annals of the New York Academy of Sciences, 1223, 1-38.
DOI:10.1111/j.1749-6632.2011.06004.x

Lambert, T.D., Malcolm, J.R., y Zimmerman, B.L. (2005). Variation in Small Mammal Species Richness by Trap Height and Trap Type in Southeastern Amazonia. Journal of Mammalogy, 86, 982-990. DOI:10.1644/15451542(2005)86[982:vismsr]2.0.co;2

Lande, R. (1995). Mutation and Conservation. Conservation Biology, 9, 782-791. DOI:10.1046/j.15231739.1995.09040782.x

May, R.M. (1988). Control of feline delinquency. Nature, 332, 392-393. DOI:10.1038/332392a0

Montoya, J., Ruiz, D.M., Andrade, G., Matallana, C., Díaz Timoté, J.J., Azcárate, J., y Areiza, A. (2018). Visión integral para la gestión de las áreas protegidas urbanas en Colombia. Biodiversidad en la Práctica, 3(1), 51-73. Retrieved from http://revistas.humboldt.org.co/ index.php/BEP/article/view/549

Morales-Jiménez, A.L., Link A., y Stevenson, P. (2008). Saguinus leucopus (Silvery-brown Tamarin). The IUCN Red List of Threatened Species 2008: e.T19819A9019454. https://www.iucnredlist.org/species/19819/9019454. Accessed 25 Jan 2020

Ochoa, G.J., Bevilacqua, M., y García, F. (2005). Evaluación ecológica rápida de las comunidades de mamíferos en cinco localidades del delta del Orinoco, Venezuela. Interciencia, 30, 466-475. Retrieved from http://ve.scielo.org/scielo.php?script=sci_arttext\& pid=S0378-18442005000800007

Patton, J.L., Pardiñas, U.F.J., y D'Elía, G. (Eds.). (2015). Mammals of South America, Volume 2: Rodents. Chicago and London: University of Chicago Press.

Proulx, G. (1988). Control of Urban Wildlife Predation by Cats Through Public Education. Environmental Conservation, 15, 358-359. DOI:10.1017/S0376892900029878

Ramírez-Chaves, H.E., y Suárez-Castro, A.F. (2014). Adiciones y cambios a la lista de mamíferos de Colombia: 500 especies registradas para el territorio nacional. Mammalogy Notes, 1, 31-34. DOI:10.47603/manovol1n2.31-34

Ramírez-Chaves, H.E., Suárez-Castro, A.F., y GonzálezMaya, J.F. (2016) Cambios recientes a la lista de los mamíferos de Colombia. Mammalogy Notes, 3, 1-9. DOI:10.47603/manovol3n1.1-9

Roemer, G.W., Gompper, M.E., y Van Valkenburgh, B. (2009). The Ecological Role of the Mammalian Mesocarnivore. BioScience, 59, 165-173. DOI:10.1525/bio.2009.59.2.9

Rovero, F., Tobler, M., y Sanderson, J. (2010). Camera trapping for inventorying terrestrial vertebrates. En: Eymann, J., Degreef, J., Häuser, C., Monje, J.C., Samyn, Y., y Vanden Spiegel, D. (eds.) Manual on field recording techniques and protocols for all taxa biodiversity inventories and monitoring (pp 100-128). ABC Taxa, Brussels.

SiB: Sistema de Información sobre Biodiversidad de Colombia. (2020). Biodiversidad en cifras. Retrieved from https:// cifras.biodiversidad.co/. Accessed 21 Jan 2020

Sikes, R.S., y Gannon, W.L. (2011). Guidelines of the American Society of Mammalogists for the use of wild mammals in research. Journal of Mammalogy, 92, 235-253. DOI:10.1644/10-mamm-f-355.1

Sánchez-Londoño, J.D., Marín-C, D., Botero Cañola, S., y Solari, S. (2014). Imama. Mamíferos silvestres del Valle 
de Aburrá. Área Metropolitana del Valle de Aburrá, Corantioquia, Universidad de Antioquia. Medellín

Solari, S., Muñoz-Saba, Y., Rodríguez-Mahecha, J.V., Defler, T.R., Ramírez-Chaves, H.E., y Trujillo, F. (2013). Riqueza, endemismo y conservación de los mamíferos de Colombia. Mastozoología Neotropical, 20(2), 301-365. https://www.redalyc.org/pdf/457/45729294008.pdf

Soto-Calderón, I.D., Acevedo-Garcés, Y.A., Álvarez-Cardona, J., Hernández-Castro, C., y García-Montoya, G.M. (2016). Physiological and parasitological implications of living in a city: the case of the white-footed tamarin (Saguinus leucopus). American Journal of Primatology, 78(12), 1272-1281. DOI:10.1002/ajp.22581

Voss, R.S., y Emmons, L. (1996). Mammalian diversity in Neotropical lowland rainforests: a preliminary assessment. Bulletin of the American Museum of Natural History, 230, 1-115. http://hdl.handle.net/2246/1671 